\title{
Estructura del arbolado y caracterización dasométrica de la segunda sección del Bosque de Chapultepec
}

\author{
Dasometric characterization and structure of tree stands \\ at Chapultepec Forest second section
}

\author{
Héctor M. Benavides Meza1 y Diana Young Fernández Grandizo1
}

\begin{abstract}
RESUMEN
El Bosque de Chapultepec es el área verde urbana (AVU) de mayor extensión y con más visitantes de la Ciudad de México, razón por la cual su protección y conservación tiene gran relevancia por los servicios ambientales y beneficios antropocéntricos que genera. No obstante su trascendencia, hasta la fecha no se ha estudiado la estructura horizontal ni se han generado indicadores de su masa arbolada. Esta situación es extensiva al resto de las AVU de la ciudad y del país y solo se han realizado ocasionalmente estudios florísticos o diagnósticos físico-sanitarios. Se determinaron las características dendrométricas y dasométricas del arbolado en la $2^{\mathrm{a}}$ sección, con base en información registrada durante el inventario total de sus árboles, complementada con la obtenida en 111 sitios de muestreo de $500 \mathrm{~m}^{2}$. Se determinó una densidad promedio de árboles de 295/ha; un valor promedio de cobertura de copa por árbol de $27,19 \mathrm{~m}^{2}$ y la cubierta del dosel excede $80 \%$ de la superficie; mientras que el valor promedio de área basal por árbol fue de $0,10 \mathrm{~m}^{2}$ y los fustes ocupan $0,32 \%$ de dicha superficie. Los datos de este trabajo servirán como un indicador básico para la $2^{\mathrm{a}}$ sección y una referencia para futuros trabajos de investigación y caracterización de masas arboladas urbanas, lo cual es fundamental para sustentar el manejo que requiere el bosque urbano de la Ciudad de México.
\end{abstract}

PALABRAS CLAVE:

Arbolado urbano, área basal, áreas verdes urbanas, Ciudad de México, dasonomía urbana.

\begin{abstract}
Chapultepec Forest is the largest and most visited urban green area (UGA) of Mexico City and for these reasons, the protection and conservation of this place is a relevant issue to maintain the generation of the environmental services and benefits that it provides. Despite the importance of this area, there is a lack of information about the horizontal structure and indicators of the tree stands located in this place, as well as for the rest of the UGA of the city and the country, although some floristic or sanitary conditions studies had been done in some of them. The aim of this paper was to determine the dendrological and dasometric characteristics of the stands at the 2nd section of Chapultepec Forest, according with the information obtained from a total tree inventory, complemented with 111 sampling sites of $500 \mathrm{~m}^{2}$. The estimated mean of trees per site was $295 /$ ha, the mean crown cover value was $27,19 \mathrm{~m}^{2}$ and the canopy cover in the area exceeds $80 \%$ of the total surface. The mean basal area was $0,10 \mathrm{~m}^{2}$ and the stems occupied $0,32 \%$ of the total surface. This data will provide basic indicators for the 2 nd section tree stands and a reference for future research projects and characterization of UGA in the city. In addition, the information is basic to sustain the management that requires the urban forest of Mexico City.
\end{abstract}

KEY WORDS:

Urban trees, basal area, urban green areas, Mexico City, urban forestry.

1 CENID-COMEF, Instituto Nacional de Investigaciones Forestales, Agrícolas y Pecuarias. Av. Progreso núm. 5, Barrio de Santa Catarina, Del. Coyoacán, C.P. 04010. México, D.F. C.e.: benavides.hector@inifap.gob.mx. 


\section{INTRODUCCIÓN}

El bosque urbano está conformado por los árboles, arbustos y vegetación herbácea asociada a estos, que se encuentran dentro de los límites de la ciudad. Lo compone tanto el arbolado de alineación en banquetas de calles, avenidas y camellones, como las áreas verdes urbanas (AVU), las cuales son espacios públicos bajo la responsabilidad de los gobiernos municipales o delegacionales. También es conveniente incluir en las AVU los derechos de vía, cementerios, barrancas y riberas de ríos o arroyos (Hitchings, 1981; Benavides, 1989); así como las grandes áreas arboladas, naturales o inducidas, que se encuentren dentro de la mancha urbana y que comúnmente se les denominan bosques (Chapultepec, San Juan de Aragón y Tlalpan, como ejemplos para la Ciudad de México).

El bosque urbano interactúa con el resto de los componentes bióticos y abióticos del ecosistema urbano y contribuye al mejoramiento de la calidad de vida de la población y del ambiente citadino, razón por la cual es importante conocer las características y condiciones de las masas arboladas que lo conforman, particularmente en aquellas AVU sujetas a una fuerte presión de uso como es el caso del Bosque de Chapultepec.

Las masas arboladas urbanas, al igual que las forestales, están compuestas por diferentes estratos, los cuales pueden ser ubicados en una distribución vertical (desde el rastrero hasta el arbóreo superior), asimismo, una distribución horizontal de gran trascendencia, pues es un indicador de la respuesta del arbolado a las condiciones en que se ha desarrollado desde su plantación, comúnmente antropogénica, pero influida en forma decisiva por la presión humana, los factores ambientales y las actividades de mantenimiento que se aplicaron en dicho lugar (Sacksteder y Gerhold, 1979; Smiley y Baker, 1988).

\section{Condiciones de las áreas verdes}

Cuando una AVU recibe un número considerable de visitantes y no se realizan las acciones de mantenimiento acordes a esa situación, el deterioro que sufre el arbolado y el sitio puede llegar a ser difícil de revertir y, como consecuencia, se afectan los beneficios y servicios ambientales que se derivan.

Las AVU de la Ciudad de México y del resto del país no cuentan con programas de manejo, por lo que carecen de un documento rector que tutele $y$ oriente las actividades de mantenimiento y mejoramiento necesarias. Ejemplo de lo anterior es la presencia de masas arboladas con especies altamente representadas o con elevadas densidades de plantación, que provocan que los árboles presenten deformaciones por la búsqueda de luz o bien estén suprimidos o debilitados por la falta de un abasto suficiente de agua y nutrientes minerales, lo que los predispone a una infestación por agentes bióticos nocivos.

\section{Bosque de Chapultepec}

Los primeros vestigios que se tienen en el lugar provienen del pueblo tolteca (1280), sin embargo, fueron los mexicas quienes incorporaron Chapultepec al desarrollo de la majestuosa Ciudad de México -Tenochtitlan, pues sus manantiales fueron utilizados como abasto de agua y sus zonas boscosas como área recreativa y coto de caza para los emperadores de esa ciudad estado (Nattal, 1923; Tovar, 1982).

En la actualidad el Bosque de Chapultepec está conformado por tres 
secciones que, en su conjunto, tienen una superficie de 686,01 ha, lo que la hace el AVU de mayor extensión en la Ciudad de México y su área metropolitana. Es un sitio de gran trascendencia social, cultural, recreativa y ambiental, por lo que es uno de los parques urbanos más visitados del mundo.

La importancia meso-climática del bosque es de gran trascendencia pues, como lo refiere Jáuregui (1975), la temperatura en el área del bosque es menor, debido a la evaporación proveniente de los lagos y la evapotranspiración de las plantas y suelos, que ocasiona este contraste térmico entre el bosque y el área urbana circunvecina.

\section{Segunda sección del Bosque de Chapultepec}

La $2^{a}$ sección se inauguró el 24 de octubre de 1962 y se localiza al poniente del Anillo Periférico y al norte de la Avenida Constituyentes. Su diseño urbanístico fue realizado por el arquitecto Leónides Guadarrama y ocupa una superficie un poco mayor a 168 ha (Tovar, 1982; Hernández, 1999). En esta sección se encuentran los museos de Historia Natural, Papalote Museo del Niño y Tecnológico de la CFE; así como espacios recreativos de gran tradición como La Feria y diversas fuentes escultóricas, entre las que destaca la de Tláloc realizada por Diego Rivera y que se ubica en las cercanías del cárcamo que fue construido para recibir el agua proveniente de Lerma, Estado de México (Hernández, 1999; GDF, 2006).

El Gobierno del Distrito Federal (2006) reportó que las principales especies de árboles que se encuentran en la $2^{\mathrm{a}}$ sección son Eucalyptus camaldulensis Dehnh. (eucalipto rojo), Fraxinus uhdei (Wenz.) Lingelsh. (fresno) y Ligustrum
Iucidum W.T. Aiton (trueno lila), los cuales se presentan en más de $80 \%$ de las áreas verdes; sin embargo, no se menciona en ese trabajo cómo se realizó el estudio ni la frecuencia de los individuos.

En fecha reciente, la Dirección del Bosque de Chapultepec solicitó al Instituto Nacional de Investigaciones Forestales, Agrícolas y Pecuarias (INIFAP), la realización de varios proyectos en la zona, para lo cual fue fundamental la colaboración del Fideicomiso Pro-Bosque de Chapultepec. Entre ellos destaca la caracterización y diagnóstico de la $2^{a}$ sección, así como el inventario total de su arbolado, en el cual fue posible registrar las características, condiciones, especies y ubicación de los 38825 individuos de tipo leñoso que se encontraron en este lugar (32 211 árboles, 3142 arbustos y 3472 arbolitos) (INIFAP-DBCh, 2009).

\section{Caracterización de los bosques}

El conocimiento de la estructura, conformación, distribución y desarrollo de las masas arboladas es importante, pues ayuda a clasificar y entender la influencia de los procesos que se han presentado en las mismas. Para llevar a cabo lo anterior se necesita definir la composición de especies, densidad de los árboles, estratos presentes e incluso la estimación del crecimiento potencial en las localidades donde crecen (Hocker, 1984).

El primer paso en la evaluación de una comunidad arbórea es su descripción cualitativa, la cual es muy valiosa e informativa, pero es básicamente subjetiva y por lo mismo está sujeta a las habilidades y sesgo del observador (Spurr, 1964). Con el fin de evitar esta situación, la caracterización de la estructura del estrato arbóreo debe basarse en índices cuantitativos que permitan analizar objetivamente las influencias antropogénicas 
que se ejercen o los procesos de sucesión natural que ocurren en los ecosistemas forestales (Aguirre, 2002).

La caracterización de la estructura es una condicionante básica para tomar decisiones sobre el manejo de los recursos forestales, tanto en localidades bajo aprovechamiento o uso, como en áreas naturales protegidas (Corral et al., 2005). El bosque urbano, al igual que las masas forestales, requiere del conocimiento de sus características, composición, densidad, estado físico y estado sanitario, con el fin de determinar su situación y las acciones de mantenimiento necesarias, así como programar y presupuestar los recursos financieros, humanos y materiales que se requieren para su conservación, mejoramiento y en caso de ser posible, fundamentar su manejo.

\section{Densidad del rodal}

El desarrollo de una estructura vertical y horizontal en el bosque es resultado de la competencia entre las especies vegetales, las cuales refieren la ocupación en superficie de los árboles sobre el suelo (Corvalán y Hernández, 2006). La diferencia fundamental entre un bosque bajo manejo o sin éste es el control de la densidad (Becerra, 1986). Spurr y Barnes (1980) definen la densidad como la cantidad de árboles o área basal por unidad de área, pero debe considerarse como un indicador del grado en que los árboles ocupan el área de un lugar en un momento dado y permite representar parcialmente y de manera sencilla la estructura de los rodales (Zepeda, 1984).

La densidad puede ser determinada por medio de varios parámetros entre los que destacan el número de árboles, área del fuste, área basal, diámetro normal, volumen, altura dominante, etc. Dichos parámetros permiten la elaboración de índices con los cuales se puede deter- minar el grado de ocupación de los árboles en un área específica. Ejemplo de ellos son los índices de densidad basados en el espacio de crecimiento de los individuos (Becerra, 1986) y puede evaluarse en términos de área basal o cobertura del dosel (Corvalán y Hernández, 2006).

El control de la densidad es muy importante, ya que influye en el crecimiento de los árboles. En los rodales coetáneos la competencia por la luz, la humedad y los nutrientes depende en gran medida de la densidad de los árboles en ese sitio (Spurr y Barnes, 1980). Estos estudios han confirmado que existe una relación estrecha entre el tamaño de los individuos y su número por unidad de superficie en diferentes situaciones competitivas y es independiente de la edad y de la calidad del sitio, lo cual ofrece una base de la cual se desprende el conocimiento de las interacciones competitivas entre los individuos de una población (Márquez y Álvarez, 1995).

Si bien se ha generado este tipo de información para especies forestales como por ejemplo Pinus patula Schl. et Cham. (Becerra, 1986), Pinus cooperi var. ornelasi Martínez (Márquez y Álvarez, 1995), Pinus hartwegii Lindl. y Pinus pseudostrobus Lindl. (Zepeda y Villarreal, 1987 y Aguirre et al., 1994), este tipo de estudios no han sido aún realizados en masas arboladas urbanas de la Ciudad de México e incluso de otras ciudades de la República Mexicana.

Una razón de lo anterior puede estar relacionada con el hecho de que la definición de la densidad óptima en este tipo de masas arboladas no es una tarea sencilla, ya que se deben de tomar en cuenta múltiples condiciones bióticas, abióticas y de uso (sociales), pero indudablemente, es conveniente generar una referencia que permita orientar las actividades que 
desarrollan los encargados operativos de las $A V U$, especialmente cuando no se cuenta con planes de manejo.

\section{OBJETIVOS}

Determinar las características dendrométricas y dasométricas del arbolado y de la masa arbórea de la $2^{a}$ sección del Bosque de Chapultepec.

Determinar los indicadores básicos respecto al número de árboles y su densidad para las diferentes zonas que conforman la $2^{a}$ sección del Bosque de Chapultepec.

\section{METODOLOGÍA}

\section{Área de estudio}

El Bosque de Chapultepec se localiza al poniente del Distrito Federal en la Delegación Política Miguel Hidalgo (Casasola, 2006; GDF, 2006) (Fig. 1); aunque no depende administrativamente de ésta. Se encuentra entre los paralelos $19^{\circ} 23^{\prime} 40^{\prime \prime}$ y $19^{\circ} 25^{\prime} 45^{\prime \prime}$ de latitud norte y los meridianos 99'10'40" y 99 $14^{\circ} 15^{\prime \prime}$ de longitud oeste (PUEC-UNAM, 2002) y se presenta una diferencia de altitud en su relieve que va de los $2250 \mathrm{msnm}$ en la $1^{\mathrm{a}}$ sección, a más de $2400 \mathrm{msnm}$ en la parte más alta de la $3^{a}$ sección (Molina, 1979). El clima en el lugar se caracteriza por una precipitación abundante en la estación lluviosa $(700 \mathrm{~mm}$ a

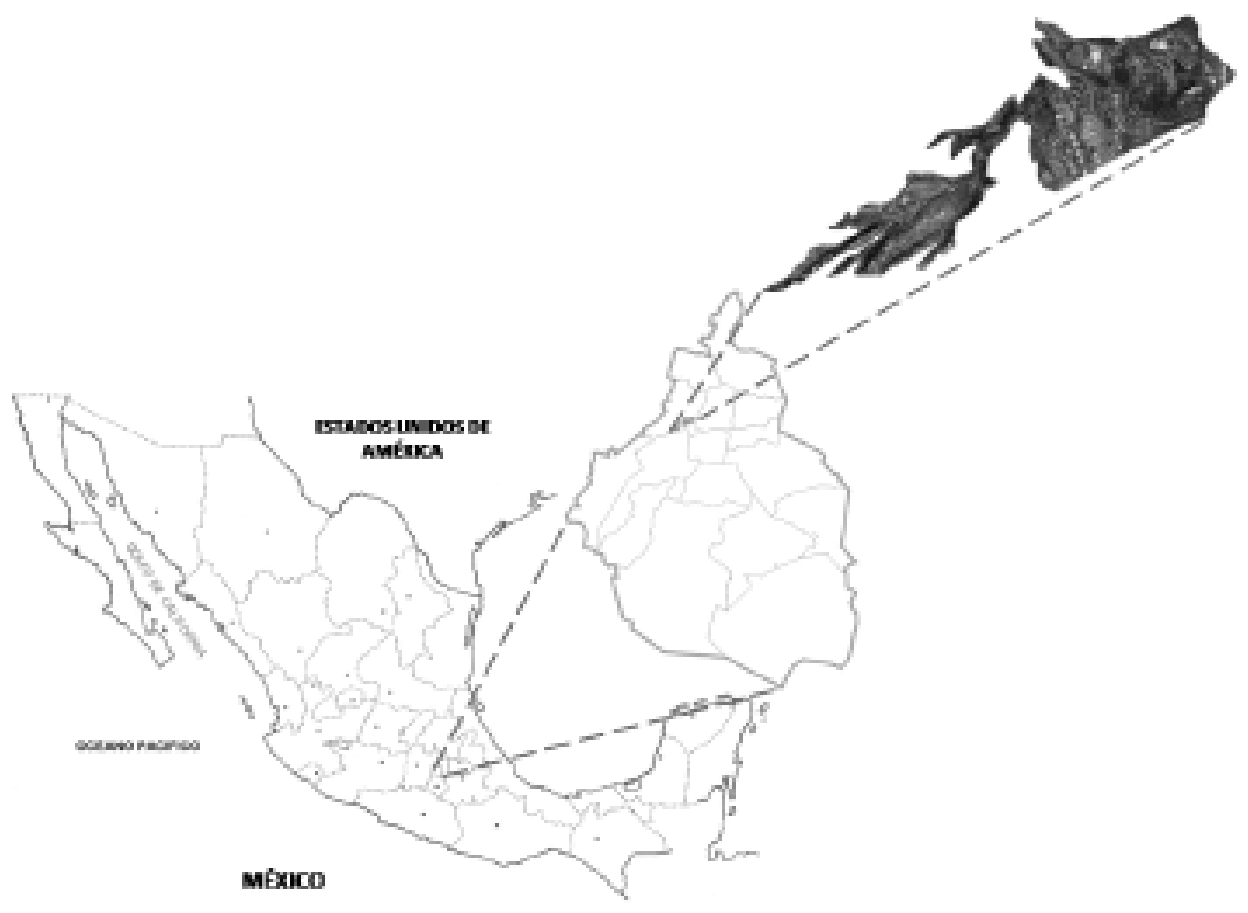

Figura 1. Ubicación del Bosque de Chapultepec en la Delegación Miguel Hidalgo del Distrito Federal, México. 
$1100 \mathrm{~mm}$ ), que corresponde a la zona de los lomeríos del borde poniente y sur de la ciudad (PUEC-UNAM, 2002). De acuerdo con Conabio (1997), el clima se clasifica como $C(w 1)(w)\left(i^{\prime}\right) g$, es decir, templado subhúmedo con lluvias en verano y temperatura media anual entre $12{ }^{\circ} \mathrm{C}$ y $18^{\circ} \mathrm{C}$, con poca oscilación anual entre las temperaturas medias del mes más frío y del mes más caliente (entre $5{ }^{\circ} \mathrm{C}$ y $7{ }^{\circ} \mathrm{C}$ ) y una marcha anual de la temperatura tipo Ganges. Los veranos en Chapultepec son frescos y largos, el mes más cálido se presenta antes de junio. Su índice de humedad (P/T) está entre 43,2 y 55,0 y la cantidad de lluvia es escasa en invierno (menor de 5\%).

\section{Inventario y registro del arbolado}

La zonificación que se utilizó en el presente trabajo corresponde a la empleada en el inventario del arbolado de la $2^{\mathrm{a}}$ sección del Bosque de Chapultepec, en el cual se dividió en zonas y subzonas para facilitar el muestreo y registro de los árboles (Fig. 2). La descripción y límites de las mismas se refieren en el informe final de dicho proyecto (INIFAP-DBCh, 2009) y en la figura 2 se presenta su ubicación.

Los árboles y arbustos de la $2^{a}$ sección fueron inventariados de acuerdo con la metodología utilizada en el Programa de Investigación de Dasonomía

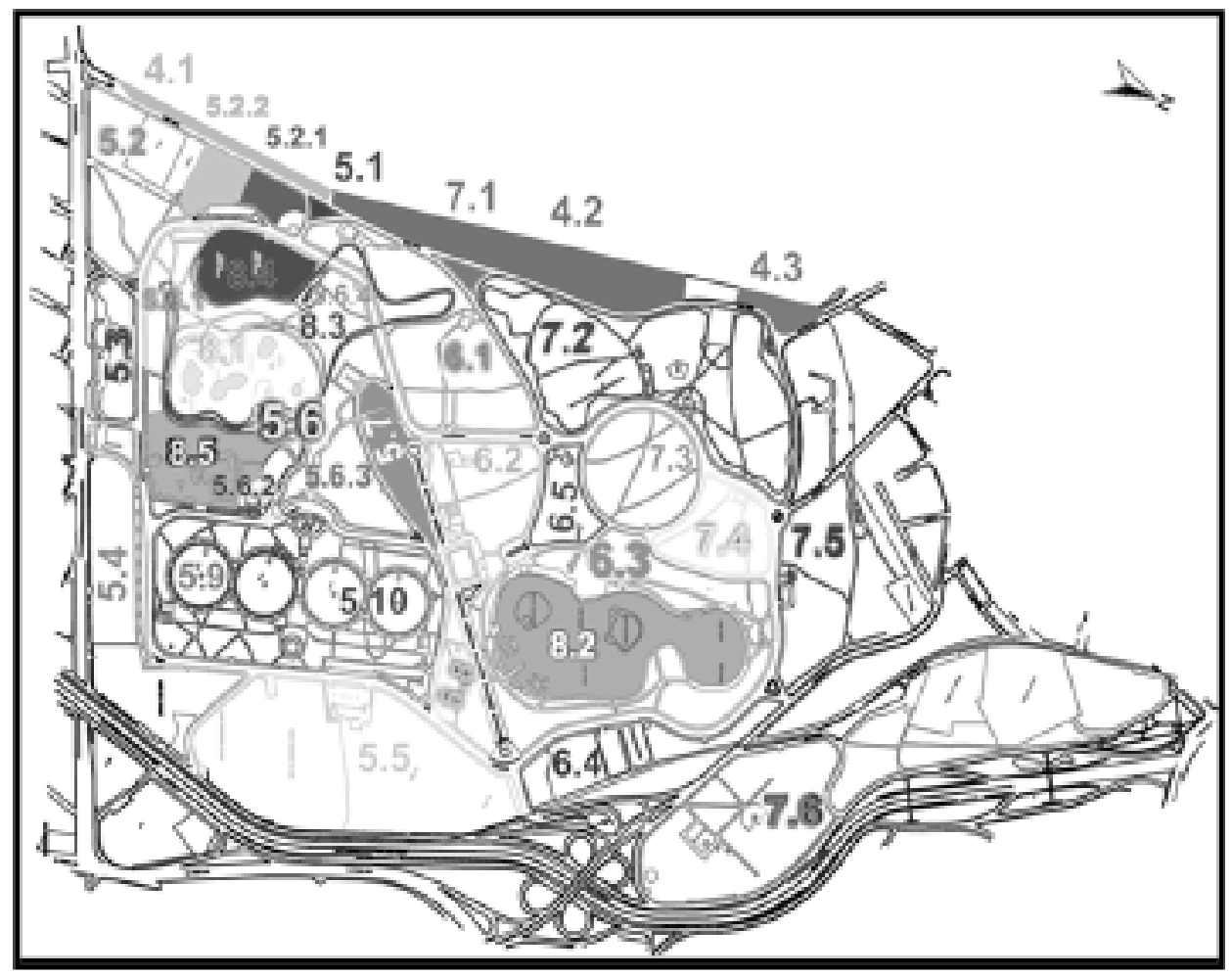

Figura 2. Zonificación de la segunda sección del Bosque de Chapultepec para la realización del inventario total de su arbolado. 
Urbana del INIFAP, que por no ser parte medular de este reporte no se describe en extenso; pero entre las características y variables determinadas en cada planta se encuentran: especie, etapa de edad, datos dendrométricos (altura, diámetro normal y basal), cobertura de copa, estado físico del tronco y de la copa, así como la condición sanitaria del tronco y de la copa. Los árboles fueron etiquetados y se generó una base de datos y un sistema de consulta para su utilización por parte del personal de la Dirección del Bosque de Chapultepec.

\section{Trabajo de campo complementario}

En adición al inventario y con el propósito de estimar en forma confiable los diferentes parámetros de interés relacionados con este componente del proyecto, se definió un diseño de muestreo sistemático que consistió en una retícula de puntos equidistantes $100 \mathrm{~m}$ y ubicados de acuerdo a una imagen georreferenciada de la $2^{\mathrm{a}}$ sección del Bosque de Chapultepec (Fig. 3).

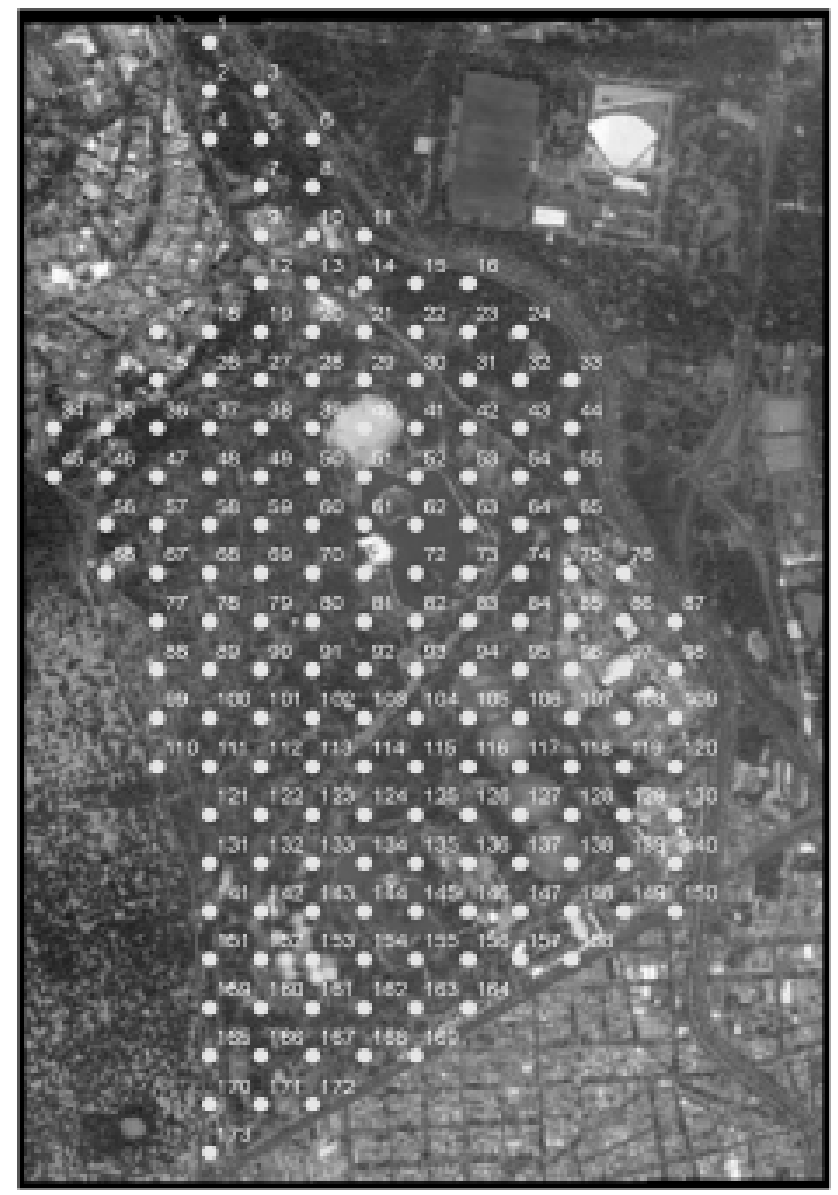

Figura 3. Retícula de puntos de muestreo (sitios) en la $2^{a}$ sección del Bosque de Chapultepec. 
Los puntos estaban referidos por coordenadas UTM y marcaban el centro de los sitios de muestreo, los cuales tenían una forma circular de $12,6 \mathrm{~m}$ de radio $\left(500 \mathrm{~m}^{2}\right)$ y fueron localizados mediante un geoposicionador. De los 173 sitios posibles de muestrear solo se evaluaron 111, debido a que varios se ubicaron en edificaciones, vialidades, fuentes $o$ instalaciones recreativas $y$ culturales. En la tabla 1 se presenta una relación del número de sitios muestreados por zona y la superficie de cada una de ellas, aunque cabe aclarar que los puntos ubicados en la zona 8, están considerados dentro de la 5 (Fig. 2).

Tabla 1. Superficie de cada zona en la $2^{a}$ sección del Bosque de Chapultepec

y número de sitios de muestreo.

\begin{tabular}{ccc}
\hline Zona & $\begin{array}{c}\text { Superficie } \\
\text { por zona (ha) }\end{array}$ & $\begin{array}{c}\text { Núm. de sitios } \\
\text { muestreados }\end{array}$ \\
\hline
\end{tabular}

\begin{tabular}{lcc}
4 & 5,3 & 5 \\
5 & 39,6 & 46 \\
6 & 22,5 & 19 \\
7 & 38,5 & 41 \\
Total & 105,9 & 111 \\
\hline
\end{tabular}

El área exclusivamente arbolada en la $2^{\mathrm{a}}$ sección del Bosque de Chapultepec, es decir, la que no está ocupada por museos, escuelas, vialidades y otras construcciones fue estimada en 1'059168 m² (105,9 ha), por lo que la superficie de los 111 sitios de muestreo (55 $500 \mathrm{~m}^{2}$ o 5,55 ha), representa $5,24 \%$ de dicha superficie.

Los datos de campo se obtuvieron de diciembre de 2008 a mayo de 2009 y la información registrada a nivel de unidad de muestreo fue la siguiente: número de sitio, número de árbol central (de acuerdo con el que le había sido asignado en el inventario), fecha, coordenadas UTM y especie arbórea preponderante en el sitio. Todos los árboles que se encontraban dentro del radio se registraron y se complementó la información recabada durante el inventario con datos relativos a la evidencia de ataque de plagas y/o enfermedades, así como la cobertura de copa, la cual se midió con cinta métrica en dos dimensiones que formaban un ángulo de $90^{\circ}$ y siempre de línea de goteo a línea de goteo.

Con los datos recabados en campo se hizo el proceso pertinente de los datos para determinar el área basal $(A B)$ y la superficie ocupada por las copas (CC). La primera se refiere a la superficie ocupada por los troncos y se calculó conforme a la ecuación 1 , que permite determinar el área basal $(A B)$ en $\mathrm{m}^{2}$ a partir del diámetro basal $(D B)$ en $\mathrm{cm}$ :

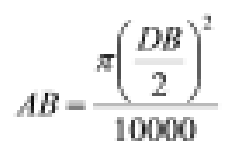

La cobertura de copa es definida por FONAM (2006) como la proyección ortogonal de las copas de los árboles sobre la superficie, es decir, hace referencia a la superficie del suelo que es cubierta por individuos de porte arbóreo de una o todas las especies. Esta variable dendrométrica se obtuvo a partir del promedio de los segmentos derivados de las dos dimensiones registradas, el cual se consideró entonces como el diámetro de la copa (Prom C) y se obtuvo el radio (Ec. 2).

$$
C C=\pi\left(\frac{\operatorname{Prom} C}{2}\right)^{2}
$$

Con base en lo anterior, se estimó el área ocupada tanto por el fuste como por las copas de los árboles en cada zona y por extensión para la $2^{a}$ sección del Bosque de Chapultepec. Cabe destacar que en estos cálculos no se incluyó la información de los árboles pequeños (diámetro normal menor a $5 \mathrm{~cm}$ y altura menor a 2,5 
m) debido a que este tipo de individuos son muy susceptibles a ser eliminados por vandalismo o por cualquier factor abiótico que ocasiona su rápida pérdida de vigor y muerte, que se relaciona con la dinámica del bosque urbano y que conlleva una gran fluctuación en su número en el corto plazo $\mathrm{y}$, por ende, su contribución es poco representativa para los valores dasométricos.

\section{RESULTADOS}

\section{Número de árboles}

En los 111 sitios de muestreo se registraron 1843 árboles pertenecientes a 41 especies, aunque se encontraron diferencias en el número de individuos por sitio de acuerdo con la zona. Los mayores valores se presentaron en las zonas 5 y 7 (16 y 18, respectivamente) y el promedio de árboles por sitio dependiendo de la zona fue de 9 a 18, mientras que el promedio general para la $2^{a}$ sección fue de 15 árboles por sitio (Tabla 2).

Con base en estos datos, se realizó la estimación del número de árboles promedio por cada $100 \mathrm{~m}^{2}$ y por hectárea en cada zona (3,15 y 295 árboles, respectivamente). En la tabla 2 se presenta también el número de árboles estimados por zona, con base en los datos promedio y la superficie de cada una, así como el número de individuos que fue registrado durante el inventario y la diferencia entre estimación e inventario. En las zonas 5 y 7 se estimó un número mayor de árboles que los que fueron registrados en el inventario, mientras que en las zonas 4 y 6 se presentó una situación contraria, pues se registraron más árboles en el inventario que el obtenido con base en las estimaciones, lo que podría indicar una sobreplantación en dichas zonas.

\section{Distancia promedio al árbol central}

En la tabla 3 se muestran los resultados de la distancia promedio y distancia mínima al árbol central por zona, de los cuales el menor valor se registró en la zona 6 y el promedio general para la $2^{a}$ sección que fue de $4,36 \mathrm{~m}$; aunque cabe mencionar que en algunos sitios este valor llegó a ser de 0,3 m.

En relación con lo anterior, es importante mencionar que aún cuando el número de árboles registrados en la zona 4 fue mayor que el estimado, la distancia promedio entre árboles fue más amplia de todas las zonas, lo que indica probablemente una distribución por grupos.

\section{Cobertura de copa}

En cada sitio de muestreo de $500 \mathrm{~m}^{2}$, el área ocupada por las copas de los árboles fue superior a $400 \mathrm{~m}^{2}$, es decir, una cobertura de copas del $84,8 \%$. Con el fin de contar con parámetros de mayor facilidad de aplicación, se estimó para cada zona la cobertura promedio por cada $100 \mathrm{~m}^{2}$, su extrapolación por hectárea $\left(8482,7 \mathrm{~m}^{2}\right)$ y el promedio general para la $2^{\mathrm{a}}$ sección del Bosque de Chapultepec (Tabla 4). Asimismo, se determinó el índice de cobertura por zona (superficie cubierta por las copas de los árboles con respecto a la superficie del lugar), que puede tener valores que van de 0 (ausencia de dosel) a 1 (traslape íntegro de la superficie foliar con la del sitio). Con los datos estimados por zona, se calculó un índice promedio general para la $2^{\mathrm{a}}$ sección del Bosque de Chapultepec de 0,85 (Tabla 4). 


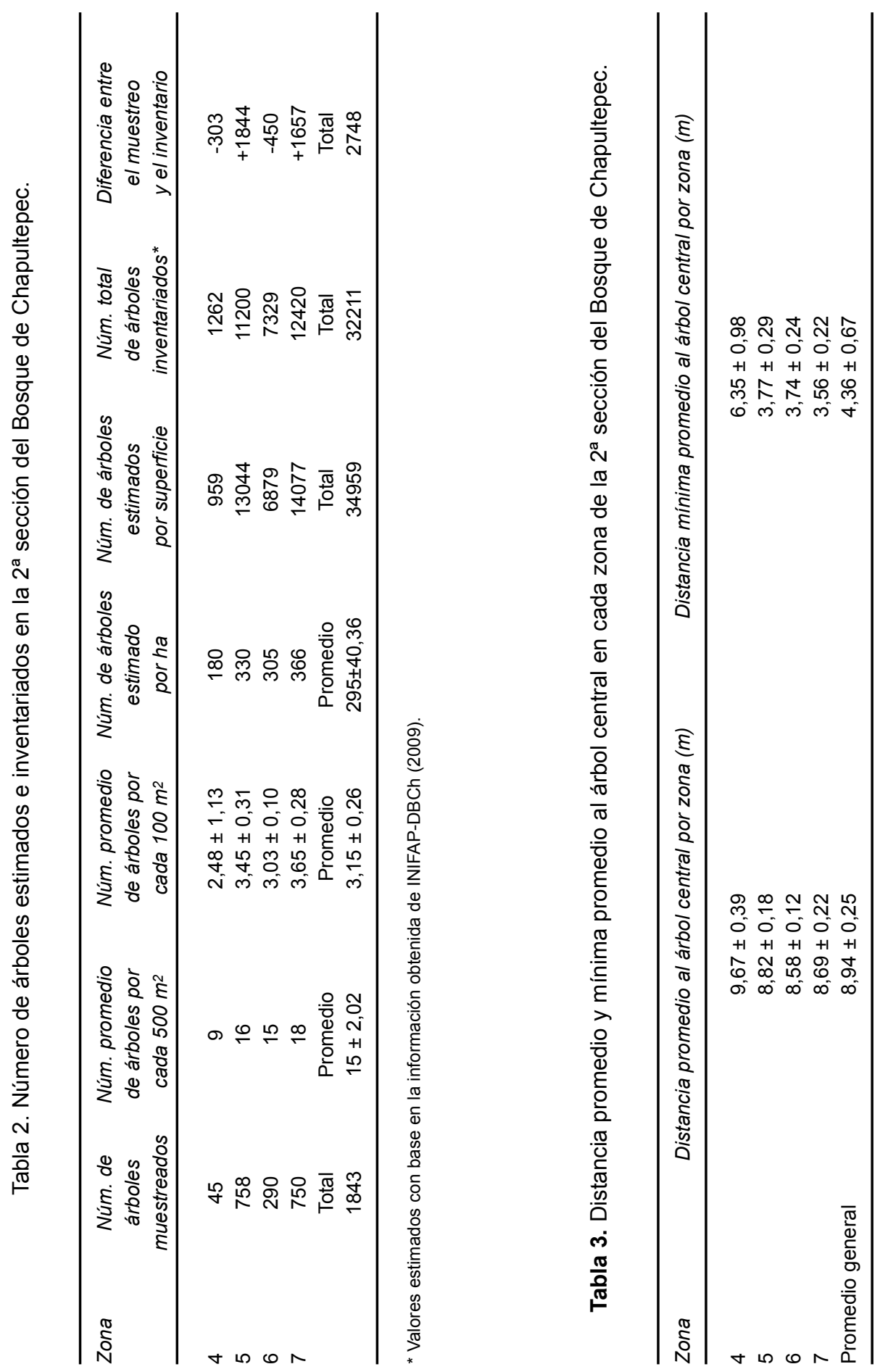


Tabla 4. Cobertura de copa para cada zona de la $2^{a}$ sección del Bosque de Chapultepec.

\begin{tabular}{lcccc}
\hline Zona & $\begin{array}{c}\text { Cobertura promedio } \\
\text { por cada } \\
500 m^{2}\left(m^{2}\right)\end{array}$ & $\begin{array}{c}\text { Cobertura } \\
\text { de copa } \\
\text { estimada por ha }\left(m^{2}\right)\end{array}$ & $\begin{array}{c}\text { Índice de cobertura } \\
\text { de copa } \\
\text { promedio por ha }\end{array}$ & $\begin{array}{c}\text { Cobertura } \\
\text { de copa } \\
\text { promedio }\left(m^{2}\right)\end{array}$ \\
\hline 4 & $408,1 \pm 213,4$ & 8161,9 & $0,82 \pm 0,43$ & $31,7 \pm 2,8$ \\
5 & $423,1 \pm 31,5$ & 8462,6 & $0,85 \pm 0,06$ & $24,3 \pm 1,7$ \\
6 & $418,5 \pm 77,6$ & 8369,7 & $0,84 \pm 0,16$ & $28,0 \pm 5,8$ \\
7 & $446,8 \pm 23,8$ & 8936,4 & $0,89 \pm 0,05$ & $24,9 \pm 1,7$ \\
Promedio general & $424,1 \pm 8,2$ & $8482,7 \pm 163,8$ & $0,85 \pm 0,02$ & $27,2 \pm 1,7$ \\
\hline
\end{tabular}

\section{Cobertura de copa promedio por árbol}

El valor promedio de cobertura de copa por individuo para cada zona de la $2^{\mathrm{a}}$ sección va de $24,3 \mathrm{~m}^{2}$ a $31,7 \mathrm{~m}^{2}$; mientras que el promedio general por árbol para la $2^{\mathrm{a}}$ sección fue de $27,2 \mathrm{~m}^{2}$ (Tabla 4). Con base en este último dato y en el número total de árboles inventariados, se calculó la superficie ocupada por el dosel en cada zona. La zona 4 presentó el menor valor, ya que tiene un área de $53250 \mathrm{~m}^{2}$ (5,33 ha), con una superficie ocupada por copas de $43462 \mathrm{~m}^{2}(4,35$ ha), lo que indica que $81,6 \%$ está ocupada por el dosel (Fig. 4). El valor calculado para la $2^{\mathrm{a}}$ sección asciende a $875,889 \mathrm{~m}^{2}$ (87,6 ha), la cual representa $82,7 \%$ de toda su superficie.

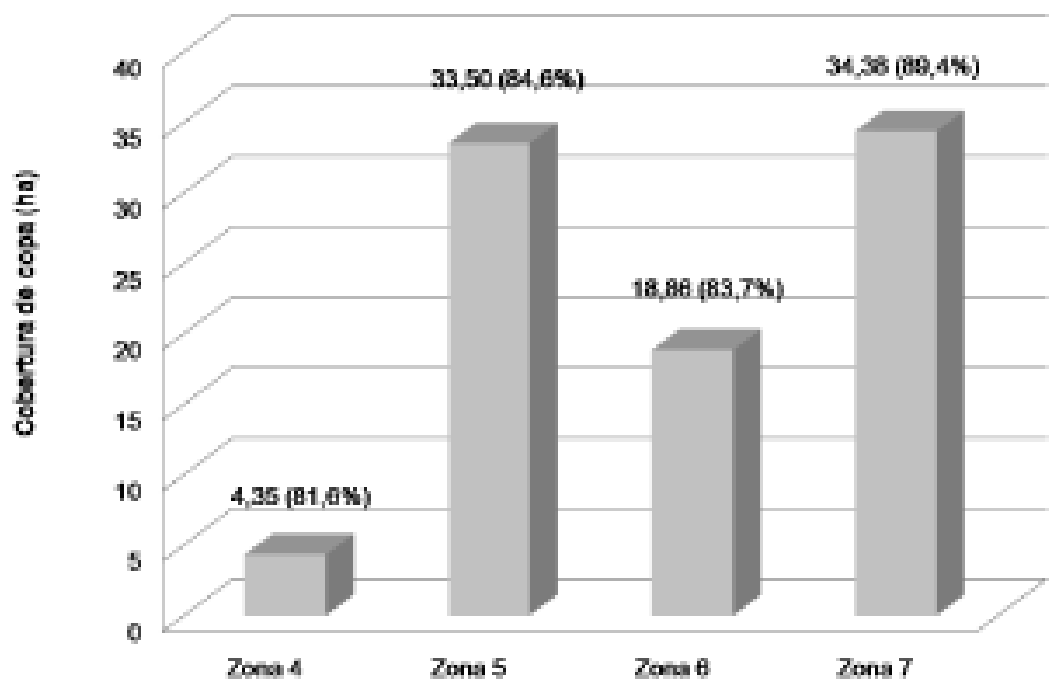

Figura 4. Cobertura de copa para cada zona de la $2^{\mathrm{a}}$ sección del Bosque de Chapultepec. 
Tabla 5. Área basal (AB) para la $2^{a}$ sección del Bosque de Chapultepec.

\begin{tabular}{lcccc}
\hline Zona & $\begin{array}{c}A B \text { promedio por } \\
\text { cada } 500 \mathrm{~m}^{2}\left(\mathrm{~m}^{2}\right)\end{array}$ & $\begin{array}{c}A B \text { estimada } \\
\text { por ha }\left(\mathrm{m}^{2}\right)\end{array}$ & $\begin{array}{c}\text { Índice de } A B \\
\text { promedio por ha }\end{array}$ & $\begin{array}{c}\text { Promedio } \\
\text { de } A B\left(\mathrm{~m}^{2}\right)\end{array}$ \\
\hline 4 & $1,44 \pm 0,45$ & 28,87 & $0,0029 \pm 0,0009$ & $0,13 \pm 0,02$ \\
5 & $1,40 \pm 0,19$ & 28,09 & $0,0028 \pm 0,0004$ & $0,10 \pm 0,01$ \\
6 & $1,65 \pm 0,22$ & 32,95 & $0,0033 \pm 0,0004$ & $0,11 \pm 0,02$ \\
7 & $1,57 \pm 0,12$ & 31,40 & $0,0031 \pm 0,0002$ & $0,09 \pm 0,005$ \\
Promedio general & $1,52 \pm 0,06$ & $30,33 \pm 1,13$ & $0,0030 \pm 0,0001$ & $0,10 \pm 0,01$ \\
\hline
\end{tabular}

\section{Área basal}

Los valores promedio de área basal registrados en los sitios de muestreo se observan en la tabla 5. De manera similar a la cobertura de copa, con dichos datos se estimó el valor por hectárea en cada zona; presentándose el menor valor en la zona 5. El promedio general para la $2^{\mathrm{a}}$ sección del Bosque de Chapultepec fue de $30,3 \mathrm{~m}^{2} / \mathrm{ha}$ (Tabla 5). Con base en estos resultados se calculó el índice de área basal para cada zona de la $2^{\mathrm{a}}$ sección, de los cuales el valor más alto se obtuvo en la zona $6(0,0033)$, que corresponde a menos del $1 \%(0,33 \%)$ de la superficie del terreno; mientras que en la zona 5 se obtuvo el valor más bajo $(0,28 \%)$. El índice promedio de área basal para la $2^{\mathrm{a}}$ sección del Bosque de Chapultepec fue de 0,0030 (Tabla 5).

\section{Área basal promedio por árbol}

Con base en los procedimientos y datos referidos anteriormente, se estimó el valor promedio de área basal por individuo arbóreo, el promedio general para la $2^{a}$ sección del Bosque de Chapultepec fue de $0,10 \mathrm{~m}^{2}$ (Tabla 5). Con base en este último dato, se calculó la superficie ocupada por los árboles inventariados en cada una de las zonas (Fig. 5).

\section{Relación área basal-cobertura de copa}

En la figura 6 se presenta la correlación entre el área basal y la cobertura de copa del arbolado ubicado en los sitios de muestreo en la $2^{a}$ sección. Los resultados muestran que $56,7 \%$ de los árboles cuentan con una cobertura de copa menor a los $20 \mathrm{~m}^{2}$ y un área basal menor a $0,1 \mathrm{~m}^{2}$; con un nivel de ajuste menor a $50 \%\left(R_{2}=0,4345\right.$ a partir de la ecuación $y=0,002 x+0,033)$. Este valor se corresponde con una probable condición de sobreplantación que influye en una deformación en el crecimiento de los individuos, lo cual repercute en copas deformadas o sobrelape de las mismas.

\section{DISCUSIÓN}

Una característica de las masas forestales bajo manejo es la manipulación de la densidad y estructura horizontal de las mismas, lo cual solo es posible cuando se tienen referencias e indicadores de ese tipo de rodales. Este tipo de información no se había generado aun para algún componente del bosque urbano de la Ciudad de México, por lo que los datos que se presentan a continuación son una primera referencia para este tipo de sitios. 


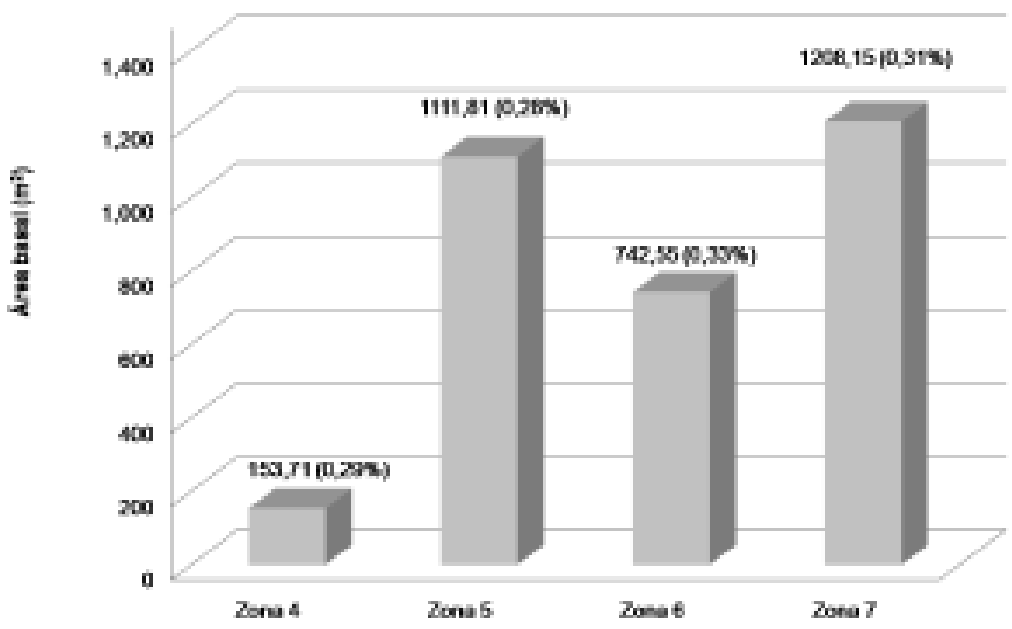

Figura 5. Proyección del área basal para las zonas que conforman la $2^{\mathrm{a}}$ sección del Bosque de Chapultepec.

El número de árboles promedio estimado para la $2^{a}$ sección del Bosque de Chapultepec fue de 295/ha, el cual se considera inferior a lo recomendable para un AVU. Este valor presenta gran disparidad entre zonas, pues en la 4 el valor promedio fue de 180 mientras que en la 7 fue de 366. Lo anterior indica una distribución no uniforme de los árboles en la $2^{\mathrm{a}}$ sección; en las zonas con mayor densidad podría estar asociado con condiciones microambientales del sitio que influyen en su sobrevivencia, asumiendo que el mantenimiento es similar.

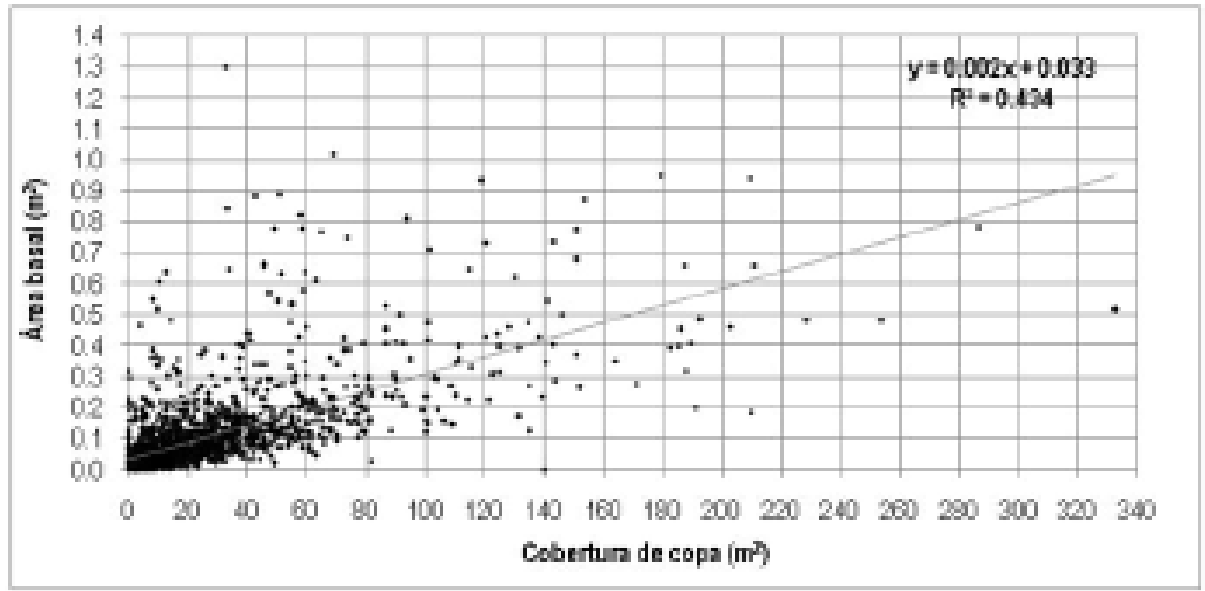

Figura 6. Relación entre el área basal y la cobertura de copa de los árboles muestreados en los sitios de la $2^{a}$ sección del Bosque de Chapultepec. 
No es posible comparar los datos registrados en este proyecto, sobre el número de árboles por superficie con ninguna otra área verde de la Ciudad de México ni del país, pues no se tiene a la fecha una referencia similar que pudiera ser utilizada. Se han realizado trabajos de tesis que abordan la evaluación de áreas verdes en diferentes lugares de la ciudad, como es el caso de Jiménez (1988) en la $1^{\text {a }}$ sección del Bosque de Chapultepec, Rojo (2006) en las delegaciones Benito Juárez y Coyoacán y Mizerit (2006) en Cuauhtémoc y Venustiano Carranza, en los cuales no se estimó o calculó esta variable, aunque reportaron valores de frecuencia y abundancia de especies con base en el muestreo realizado.

Una referencia comparativa dentro del contexto urbano es la publicada por McBride y Jacobs (1979), quienes registraron una densidad de 35 árboles por hectárea en un encinar antes de que el sitio se urbanizara. Este número se duplicó posteriormente con las prácticas de manejo e introducción de especies que se llevaron a cabo. En cambio, el proceso de urbanización en un bosque de coníferas provocó una disminución de la densidad del arbolado, al descender de 761 a 329 árboles por hectárea, aunque ciertas prácticas de plantación posterior ocasionaron que la densidad aumentara a 373 individuos, valor muy por debajo del registrado en condiciones naturales.

En forma comparativa, la densidad del arbolado que se ha publicado para zonas forestales en condiciones ambientales parecidas a las de la Ciudad de México refieren valores de 1174/ha en un bosque de oyamel conformado principalmente por Abies vejarii Martínez, Pseudotsuga menziesii (Mirb.) Franco y Quercus greggii (A. DC.) Trel. en Coahuila, México (Encina et al., 2008); mientras que Williams et al. (1996) encontraron una densidad que varió de 510/ha a 1340/ha en bosques mesófilos con diferentes grados de perturbación en las cercanías de Xalapa, Veracruz. Por su parte, Aguirre et al. (2003) refieren, para rodales de Pinus culminicola Andersen et Beaman, una cantidad de 100/ha a $780 /$ ha, lo cual es indicativo de una baja densidad debido a las condiciones climáticas extremas del sitio de estudio en el Cerro del Potosí, Nuevo León; pero en un rodal conformado por esta especie y Pinus hartwegii ubicado en una localidad cercana registraron 1113/ha. Asimismo Nájera y Hernández (2008) encontraron una densidad de 239/ha de las especies Pinus cooperi Blanco y Pinus leiophylla Schl. et Cham. al suroeste de Durango, pero estimaron que el sitio podría sostener una población de 454 individuos sin competencia entre ellos; mientras que Williams (1991) registró 960 individuos por hectárea en un bosque mesófilo de montaña en la Reserva de la Biósfera "El Triunfo", Chiapas.

Como se observa, el número de árboles por unidad de superficie es muy variable y responde principalmente a las condiciones ambientales, pero también a los factores de intervención humana, sean estas sujetas a una acción programada dentro de un programa de manejo o simple perturbación.

Con base en lo anterior, el número de árboles por hectárea para la $2^{\mathrm{a}}$ sección del Bosque de Chapultepec se ubica por debajo de lo registrado para áreas forestales, pero cercano a los datos aportados por McBride y Jacobs (1979). Esto puede estar relacionado con las condiciones limitantes que enfrenta el bosque urbano (abasto de agua, vandalismo, baja calidad del suelo, apertura inducida del espacio por debajo del dosel por cuestiones de seguridad e incluso por las actividades de recreación que practican los visitantes). No obstante, el mayor espaciamiento entre individuos permite la 
presencia de árboles en condiciones de crecimiento libre, con amplias copas y fustes relativamente altos.

\section{Distancia promedio al árbol central}

La distancia promedio entre árboles en la $2^{a}$ sección fue de $8,94 \mathrm{~m}$; mientras que la distancia mínima promedio fue de 4,36 m. En forma similar a las otras variables determinadas en la $2^{a}$ sección, existe poca información de áreas verdes en la Ciudad de México que sea comparable con estos datos y solo se cuenta con lo publicado por Mizerit (2006), quien refiere que en las áreas verdes de las delegaciones Cuauhtémoc y Venustiano Carranza la distancia fue de $3,92 \mathrm{~m}$ y $2,93 \mathrm{~m}$, respectivamente. Asimismo, Rojo (2006) registró para Benito Juárez y Coyoacán valores de $3,05 \mathrm{~m}$ y $3,08 \mathrm{~m}$, respectivamente. Alanís (2005) refiere que en relación con el espaciamiento entre árboles en una plantación urbana, se debe partir del principio de que lo que se busca es un árbol de buen porte, es decir, una estructura de copa y altura de la misma que pueda llegar a $14 \mathrm{~m}, 18 \mathrm{~m}, 22 \mathrm{~m}$ o más y con una cobertura que pueda medir de $8 \mathrm{~m}$ a $10 \mathrm{~m}$ de diámetro, por lo que se requieren distancias mínimas aceptables entre ejemplares plantados. Con base en lo anterior, es recomendable que la distancia final entre árboles maduros sea de $4 \mathrm{~m}$ a $8 \mathrm{~m}$, dependiendo de la especie, con el fin de reducir la fuerte competencia por espacio, luz, humedad y nutrientes. También se recomienda, por otra parte, dar fin a la costumbre de establecer árboles en densidades elevadas y lo que es más grave, no aplicar prácticas de aclareo, pues la resultante de estos procedimientos, con el paso del tiempo, es la presencia de árboles débiles y mal conformados, que no aportan de forma óptima los beneficios y servicios ambientales requeridos por los habitantes de las ciudades.

\section{Cobertura de copa y área basal}

Cuando el número de árboles se relacionó con la cobertura de copa y el área basal, variables de mayor utilidad para estimar el nivel o grado de ocupación del espacio disponible, se observó que el arbolado ocupa casi totalmente la superficie disponible con sus copas (8 482 $\mathrm{m}^{2} / \mathrm{ha}$ ), con un valor promedio por individuo ligeramente superior a $27 \mathrm{~m}^{2}$. Como en los casos anteriores, no se cuenta con información comparativa para el arbolado de AVU en la Ciudad de México y como referencia sólo se pueden mencionar trabajos de índole forestal, en los cuales se han publicado valores promedio de cobertura de copa de $7,9 \mathrm{~m}^{2}$ $\pm 2,4 \mathrm{~m}^{2}$ y $5,7 \mathrm{~m}^{2} \pm 4,4 \mathrm{~m}^{2}$ para individuos de Pinus cembroides Zucc. en dos rodales del sur de Nuevo León (Vallejo, 1997). Nájera y Hernández (2008) encontraron una cobertura de copa promedio por árbol de $5,21 \mathrm{~m}^{2}$ en un bosque coetáneo de $P$. cooperi y $P$. leiophylla en Durango. Arias (2005) indica que se espera la presencia de individuos con extensas copas en sitios de bajos niveles de ocupación, lo cual concuerda con los datos registrados en el presente trabajo, ya que los árboles cuentan con mayor espacio al ser menor el número de árboles por hectárea.

No obstante, los datos obtenidos en el presente trabajo para la $2^{a}$ sección deben interpretarse con cierta cautela, pues muchos árboles de este lugar son eucaliptos (principalmente E. camaldulensis), atacados por la conchuela (Glycaspis brimbiecombei Moore) y por lo mismo, la mayoría de sus copas son poco frondosas por la declinación ocasionada por el insecto chupador, lo que ha ocasionado una mayor entrada de luz hacia los estratos inferiores y al suelo. Esta situación favorece a los árboles de menor altura pues están en posibilidad de recibir una mayor radiación solar. Con base en lo 
anterior, es importante que en los árboles urbanos la cobertura de copa se relacione con lo denso u opaco de la misma y su vigor, con el fin de contar con mejores elementos de juicio para definir el valor idóneo de la misma.

En el caso del área basal los datos evidencian que en cada hectárea de la $2^{\mathrm{a}}$ sección, los troncos ocupan en promedio $30,33 \mathrm{~m}^{2}$. Si bien este valor en principio puede parecer reducido, hay que considerar que los troncos son sólo estructuras de conducción y soporte para permitir al follaje realizar las actividades fisiológicas necesarias para el desarrollo y crecimiento del árbol.

No se cuenta con datos de área basal publicados para otras AVU de la Ciudad de México o de la República Mexicana; sin embargo, el área basal registrada para un bosque templado con una temperatura media anual similar a la del Bosque de Chapultepec $\left(12{ }^{\circ} \mathrm{C}-18{ }^{\circ} \mathrm{C}\right)$, fue de 25,1 $\mathrm{m}^{2} /$ ha a $60,6 \mathrm{~m}^{2} /$ ha (Williams et al., 1996), lo cual se relaciona con una mayor precipitación $(1806 \mathrm{~mm})$ que la registrada en este parque. En el caso de bosques caducifolios conformados por especies de aprovechamiento maderable como Myracrodruon urundeuva Fr. Allem., Schinopsis brasiliensis Engl., Tabebuia impetiginosa Mart. Ex. DC., Cedrela fissilis Vell., Hymenaea courbaril L., Aspidosperma subincanum Mart. y Amburana cearensis (Fr. Allem.) A. C. Smith., se encontraron valores de densidad de área basal que van de los 23 $\mathrm{m}^{2} / \mathrm{ha}$ a los $24 \mathrm{~m}^{2} / \mathrm{ha}$ en bosques perturbados, y hasta $29,3 \mathrm{~m}^{2} / \mathrm{ha}$ en el caso de los bosques sin perturbación (Terra et al., 2004). Asimismo, el área basal publicada para una plantación comercial de eucalipto (Eucalyptus grandis Hill ex Maiden X Eucalyptus urophylla S. T. Blake), de 24 a 82 meses de edad, fue de $7,5 \mathrm{~m}^{2} / \mathrm{ha}$ a $19,45 \mathrm{~m}^{2} / \mathrm{ha}$, dependiendo de la calidad de sitio (Nogueira et al., 2005). Návar y González (2009) mencionan que en un bosque templado de encinos en Durango se registró un área basal de 11,9 m²/ha y cuando el bosque estaba conformado por pinos, el valor ascendió a 16,1 m²/ha. Encina et al. (2008) encontraron que un bosque de oyamel en Coahuila presentaba $29,69 \mathrm{~m}^{2} / \mathrm{ha}$. Williams et al. (1996) estudiaron seis sitios de bosque mesófilo en Veracruz y los resultados variaron de 25,1 $\mathrm{m}^{2} /$ ha a $60,6 \mathrm{~m}^{2} /$ ha de área basal. Un bosque templado caducifolio del este de EUA presentó un área basal de $30 \mathrm{~m} 2 / \mathrm{ha}$ para árboles con diámetro normal mayor a $10 \mathrm{~cm}$ (Held y Winstead, 1975); mientras que algunos bosques mesófilos de montaña, con dominancia de elementos templados, presentan áreas basales que también se aproximan a los $30 \mathrm{~m}^{2} / \mathrm{ha}$.

En contraste, los bosques con más especies arbóreas neotropicales presentan una mayor área basal, por ejemplo en el volcán de San Martín en Los Tuxtlas, Veracruz, el área basal es de $69,30 \mathrm{~m}^{2} / \mathrm{ha}$ en el borde del cráter y de $43,74 \mathrm{~m}^{2} /$ ha dentro del cráter (Álvarez del Castillo, 1977). En la Reserva de la Biósfera "El Triunfo", Chiapas, fue de $54,5 \mathrm{~m}^{2} / \mathrm{ha}$ (Williams, 1991) y en un bosque lluvioso de montaña del mismo estado se registraron $102 \mathrm{~m} 2 / \mathrm{ha}$ (Zuill y Lathrop, 1975).

\section{Indicadores para el manejo del bosque urbano}

La correlación que se efectuó entre el área basal y la cobertura de copa mostró un bajo ajuste debido, en principio, a la gran cantidad de especies y por ende las diferencias morfológicas de éstas. Asimismo, la sobrecompetencia entre individuos ocasiona una alteración de la forma de la copa como resultado de la sobreplantación; aunado a la deformación de las copas por podas incorrectas que afectan su estructura. No obstante, la correlación de estas variables puede ser 
de cierta utilidad como un indicador de la situación del arbolado y su densidad, especialmente cuando se tengan más datos que permitan generar indicadores más sólidos.

Es de suma importancia que se continúen realizando investigaciones de este tipo en otras AVU de la Ciudad de México y de la República Mexicana, con el fin de contar con mejores indicadores del número idóneo de árboles de acuerdo a las condiciones ambientales del sitio y que sustenten los programas de manejo que requieren.

Los datos de cobertura de copa y área basal que se han generado para la masa arbolada de la $2^{a}$ sección del Bosque de Chapultepec, proporcionan por primera vez una referencia para un AVU en México, un primer acercamiento al conocimiento dasométrico del arbolado de la Ciudad de México y una referencia paramétrica para futuros trabajos en el ámbito de la dasonomía urbana. Es importante destacar la precisión de la información generada en el presente proyecto, pues se tuvo la oportunidad de utilizar datos provenientes de un inventario total del arbolado de esta área verde, lo que le confiere a los datos una exactitud poco común, pues generalmente se obtienen con base en inventarios por muestreo.

Cuando los encargados operativos tengan a su disposición programas de manejo sutentados en indicadores confiables generados bajo criterios fundamentados en la dasonomía urbana, podrán inducir la densidad de las masas arbóreas urbanas acorde con las condiciones ambientales del área verde y por ende, contar con una base sólida que permita el manejo de las mismas e incrementar a su máximo los beneficios y servicios ambientales que proporcionan a los citadinos y al medio urbano.

\section{CONCLUSIONES}

Se presenta una gran disparidad en el número de árboles promedio por hectárea entre las zonas de la $2^{a}$ sección del Bosque de Chapultepec, que indica una distribución no uniforme del arbolado.

En las diferentes zonas de la $2^{\mathrm{a}}$ sección, la cobertura de las copas ocupan casi la totalidad de la superficie; sin embargo, la cantidad de follaje no es lo denso u opaco que sería recomendable por la declinación que presenta el arbolado.

El número de árboles y superficie ocupada por sus troncos, si bien parece un valor reducido en comparación con la superficie de la $2^{a}$ sección, permite sustentar un dosel que cubre más de $80 \%$ de esa superficie.

Los valores promedio de cobertura de copa y área basal por hectárea son ligeramente diferentes en el contexto general a los registrados para lugares de tipo forestal.

\section{AGRADECIMIENTOS}

Se agradece la colaboración del Fideicomiso Probosque de Chapultepec que financió el proyecto y a las autoridades del Bosque de Chapultepec que dieron todas las facilidades para su realización, así como a los revisores por sus atinadas sugerencias.

\section{REFERENCIAS}

Aguirre C., O.A. 2002. Índices para la caracterización de la estructura del estrato arbóreo de ecosistemas forestales. Revista Ciencia Forestal en México 27(92):5-27. 
Aguirre C., O.A., G. Vega y J. Jiménez. 1994. Guía de densidad para Pinus pseudostrobus Lindl. en Nuevo León. Memorias del Simposio y II Reunión Nacional de Silvicultura y Manejo de Recursos Forestales. $43 \mathrm{p}$.

Aguirre C., O.A., J. Jiménez P., H. Kramer y A. Akça. 2003. Análisis estructural de ecosistemas forestales en el Cerro del Potosí, Nuevo León, México. Ciencia UANL 6(2):219-225.

Alanís F., G.J. 2005. El arbolado urbano en el área metropolitana de Monterrey. Ciencia UANL 8(1):20-32.

Álvarez del Castillo, C. 1977. Estudio ecológico y florístico del cráter del volcán de San Martín Tuxtla, Ver., México. Biótica 2:3-54.

Arias, D. 2005. Morfometría del árbol en plantaciones forestales tropicales. Revista Forestal Kurú. Costa Rica 2(5):2-11.

Becerra L., F. 1986. Determinación de una guía de densidad para Pinus patula Schl. et Cham. en la región de Chignahuapan-Zacatlán, Puebla. Tesis de Maestría en Ciencias. Colegio de Postgraduados. Chapingo, Estado de México. 82 p.

Benavides M., H.M. 1989. Bosque urbano: la importancia de su investigación y correcto manejo. In: A. Villa, A. Castro y C. Nieto. eds. 1989. Memoria del Congreso Forestal Mexicano 1989. Tomo II. Toluca, Estado de México. 19 al 22 de julio de 1989. Gobierno del Estado de México y Academia Nacional de Ciencias Forestales, A.C. p:966-992.

Casasola M., M. 2006. Influencia del Bosque de Chapultepec en el clima urbano de la Ciudad de México. Tesis de Licenciatura, Carrera de Geografía, Facultad de Filosofía y Letras, UNAM. México, D. F. p:98.

Comisión Nacional para el Conocimiento y Uso de la Biodiversidad (Conabio). 1997. Carta de Climas México, a escala 1:1000000 (clasificación Köppen modificado por E. García). Comisión Nacional para Estudios de la Biodiversidad, México, D.F.

Corral R., J.J., J.J. Pérez, O.A. Aguirre C. y S. Corral R. 2005. Un análisis del efecto del aprovechamiento forestal sobre la diversidad estructural en el bosque mesófilo de montaña $\mathrm{El}$ Cielo, Tamaulipas, México. Investigación Agraria, Sistema de Recursos Forestales 14(2):217-228.

Corvalán V., P. y J. Hernández P. 2006. Densidad de rodal. Universidad de Chile, Facultad de Ciencias Forestales. Cátedra de Dasometría. Chile. 5 p. Disponible en: http://146.83.41. 79/profesor/dasometria/4.\%20DENS IDAD\%20DE\%20RODAL.pdf (28 de septiembre de 2009).

Encina D., J.A., F.J. Encina D., E. MataRocha y J. Valdés-Reyna. 2008. Aspectos estructurales, composición florística y caracterización ecológica del bosque de oyamel de la sierra de Zapalinamé, Coahuila, México. Boletín de la Sociedad Botánica de México 83:13-24. Disponible en: http://redalyc.uaemex.mx/src/inicio/ ArtPdfRed.jsp?iCve=57711109003. (15 de enero de 2010).

Fondo Nacional del Ambiente-Perú (FONAM) 2006. Propuesta final para una definición peruana de bosques a ser utilizada en proyectos de uso de la tierra, cambio de uso y silvicultura bajo el protocolo de kyoto durante el 
primer periodo de compromiso. $3 \mathrm{p}$. Disponible en: http://www.fonamperu. org/general/bosques/documentos/propuesta_final.pdf (5 de marzo de 2010).

Gobierno del Distrito Federal (GDF). 2006. Programa de rehabilitación integral del Bosque de Chapultepec. In: Dirección de Educación Ambiental-GDF, eds. 2006. Memorias. Dirección General de Bosques Urbanos y Educación Ambiental. Gobierno del Distrito Federal. Secretaría del Medio Ambiente, Dirección de Educación Ambiental. 59-113 pp. Disponible en: http://www.sma. df.gob.mx/sma/index.php?opcion=2 6\&id=406 (3 de febrero de 2009).

Held, M.E. y J.E. Winstead. 1975. Basal area and climax status in mesic forest systems. Annals of Botany 39:1147-1148.

Hernández H., A. 1999. Serie señalética para la 2a sección del Bosque de Chapultepec. Tesis Profesional. Licenciatura de Comunicación Gráfica. Escuela Nacional de Artes Plásticas, UNAM. México, D.F. $125 \mathrm{p}$.

Hitchings, D.R. 1981. Prontuario de Dasonomía Urbana. $1^{\text {a }}$ edición. Arizona State Land Dept., Forestry Division, University of Arizona \& USDA, Forest Service. Tucson, Arizona, EUA. $37 \mathrm{p}$.

Hocker, H.W. 1984. Introducción a la Biología Forestal. $1^{a}$ edición. AGT Editor, S. A. México, D.F. 446 p.

Instituto Nacional de Investigaciones Forestales Agrícolas y Pecuarias Dirección del Bosque de Chapultepec (INIFAP-DBCh). 2009. Informe final del proyecto "Diagnóstico y caracterización de la $2^{\mathrm{a}}$ sección e inventario total de su arbolado". Informe de uso Interno. s/p.

Jáuregui O., E. 1975. Microclima del Bosque de Chapultepec. Boletín del Instituto de Geografía, UNAM 6:63-72.

Jiménez S., M.R. 1988. Diagnóstico Ecológico de las Áreas Verdes de la Delegación Cuauhtémoc D.F. Tesis Profesional. Facultad de Ciencias, UNAM. México, D.F. 73 p.

Márquez L., M.A. y R. Álvarez Z. 1995. Construcción de una guía de densidad para Pinus cooperi var. ornelasi con base en la clase de copa en Durango, México. Madera y Bosques 1(2):23-36.

McBride, J.R. y D.F. Jacobs. 1979. Urban forest structure: A key to urban forest planning. California Agriculture 33(5):24. Disponible en: http://ucce. ucdavis.edu/files/repositoryfiles/ca3 305p24-62637.pdf (5 de marzo de 2010).

Mizerit T., L.H. 2006. Situación y características del arbolado en las áreas verdes de las delegaciones Cuauhtémoc y Venustiano Carranza, Distrito Federal. Tesis Profesional. Facultad de Ciencias, UNAM. México, D.F. 77 p.

Molina E.M., J.F.F. 1979. Algunos aspectos del deterioro ambiental en el Bosque de Chapultepec. Tesis Profesional, Carrera de Biología, Facultad de Ciencias, UNAM. México. 58 p.

Nájera L., J.A. y E. Hernández H. 2008. Relaciones morfométricas de un bosque coetáneo de la región de El Salto, Durango. Ra Ximhai 4(1):69-81. 
Nattal, Z. 1923. Los Jardines del Antiguo México. México Forestal 1(4):3-10.

Návar C., J. de J. y S. González E. 2009. Diversidad, estructura y productividad de bosques templados de Durango, México. Polibotánica 27:71-87.

Nogueira D., A., H. Garcia L., L. Couto y A. Carvalho F. 2005. Emprego de um modelo de crecimiento e producao em povoamentos desbastados de eucalipto. Revista Árvore 29(5): 731-739.

Programa Universitario de Estudios sobre la ciudad (PUEC-UNAM). 2002. Proyecto ejecutivo para la implementación del manejo integral y desarrollo autosostenible del Bosque de Chapultepec. DGUBUEFA-SMA. Informe de uso interno. $142 \mathrm{p}$.

Rojo N., I.A. 2006. Condiciones y características de las áreas verdes y su arbolado en las delegaciones Benito Juárez y Coyoacán, D.F. Tesis Profesional, Facultad de Ciencias, UNAM. México, D.F. 64 p.

Sacksteder, C.J. y H.D. Gerhold. 1979. A guide to urban tree inventory systems. School of Forestry Resources, Penn. State University. Research Paper 43. 52 p.

Smiley E.T. y F.A. Baker. 1988. Options in street tree inventories. Journal of Arboriculture 14(2):36-42.

Spurr, S.H. 1964. Forest Ecology. 1a edición. Ronald Press Company. Nueva York, EUA 352 p.

Spurr, S.H. y B.V. Barnes 1980. Ecología Forestal. $3^{a}$ ed. AGT editor. S.A. México. $690 \mathrm{p}$.
Terra N., A.R., A. Scariot, J. Alves da S. y A.C. Sevilha. 2004. Estimativas de área basal e uso do raloscópio de Bitterlich em amostragem de floresta estacional decidual. Ciência Florestal 14(2):169-176.

Tovar, L. 1982. Estudio descriptivo de los árboles y arbustos más comunes del Bosque de Chapultepec. Tesis Profesional, Carrera de Biología, Facultad de Ciencias, UNAM. México, D.F. 153 p.

Vallejo M., G.E. 1997. Asociación de variables dasométricas a diferentes niveles de producción de conos de pino piñonero Pinus cembroides Zucc. en el Sur de Nuevo León, México. Tesis de Maestría. Facultad de Ciencias Forestales. Subdirección de Postgrado. Universidad Autónoma de Nuevo León. 126 p.

Williams L., G. 1991. Nota sobre la estructura del estrato arbóreo del bosque mesófilo de montaña en los alrededores del campamento "El Triunfo", Chiapas. Acta Botánica Mexicana 13:1-7.

Williams L., G., I. Pérez G. y J. Tolome. 1996. El bosque mesófilo de montaña y un gradiente altitudinal en el centro de Veracruz. La Ciencia y el Hombre 8(23):149-161.

Zepeda B., E.M. 1984. Ejemplificación de tres procedimientos para caracterizar rodales por su densidad. División de Ciencias Forestales. Universidad Autónoma Chapingo. Chapingo Edo. de Mex. 57 p.

Zepeda B., E. M. y M. E. Villareal D. 1987. Guía de densidad para Pinus hartwegii Lind. de Zoquiapan, Méx. División de Ciencias Forestales. Univer- 
sidad Autónoma Chapingo. Chapingo Edo. de Mex. 52 p.

Zuill, H.A. y E.W. Lathrop. 1975. The structure and climate of a tropical montane rain forest and an associated template pine-oak-liquidambar forest in the northern highlands of Chiapas. Anales del Instituto de Biología Universidad Autónoma de México. Serie Botánica 46:73-118. trica de la segunda sección del Bosque de Chapultepec. Madera y Bosques 18(2):51-71. 